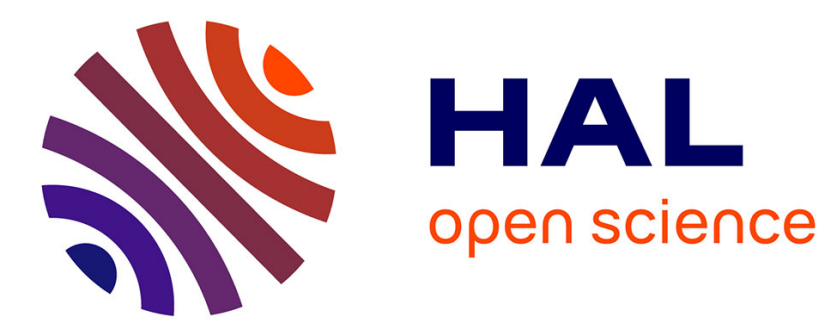

\title{
Real Time Displacement Sensor Based on Self-Mixing Interferometry
}

\author{
Antonio Luna Arriaga, Francis Bony, Thierry Bosch
}

\section{To cite this version:}

Antonio Luna Arriaga, Francis Bony, Thierry Bosch. Real Time Displacement Sensor Based on SelfMixing Interferometry. IEEE International Instrumentation and Measurement Technology Conference (I2MTC), May 2012, Graz, Austria. pp.1370-1374, 10.1109/I2MTC.2012.6229273 . hal-00716500

\section{HAL Id: hal-00716500 https://hal.science/hal-00716500}

Submitted on 10 Jul 2012

HAL is a multi-disciplinary open access archive for the deposit and dissemination of scientific research documents, whether they are published or not. The documents may come from teaching and research institutions in France or abroad, or from public or private research centers.
L'archive ouverte pluridisciplinaire HAL, est destinée au dépôt et à la diffusion de documents scientifiques de niveau recherche, publiés ou non, émanant des établissements d'enseignement et de recherche français ou étrangers, des laboratoires publics ou privés. 


\title{
Real Time Displacement Sensor Based on Self-Mixing Interferometry
}

\author{
Antonio Luna Arriaga ${ }^{1,2}$, Francis Bony ${ }^{1,2}$, Thierry Bosch ${ }^{1,2}$ \\ ${ }^{1}$ CNRS, LAAS, 7 avenue du colonel Roche, F-31400 Toulouse, France \\ ${ }^{2}$ Univ de Toulouse, INP, LAAS, F-31400 Toulouse, France \\ alunaarr@laas.fr
}

\begin{abstract}
This paper deals with the real time reconstruction of a self-mixing displacement signal under devices with limited resources. By implementing a first-order-hold interpolation used in digital-to-analog converters, the signal recovery problem has been reduced to the efficient usage of an adaptive filter with high potential of parallelism, suitable to meet hard timing constraints, low memory usage and easily integrable with other mitigation algorithms of the physical phenomena proper to laser behavior, towards the creation of a robust embedded displacement sensor.
\end{abstract}

Index Terms-Displacement measurement; optical feedback; real time systems

\section{INTRODUCTION}

The emerging optical sensors by laser self-mixing (SM) interferometry incursion in metrological fields like vibration, absolute distance, displacement, speed, and angle measurement with the premise of being a low-cost, sensitive and naturally aligned technology [1]. Due to the reduced number of components required for its setup [2], and the precision achieved with this technique [3], they offer a considerable potential for development in embedded market. The complex physical phenomena involved in SM signals generation has lead the researches to follow different paths.

As a matter of fact, for displacement measurement we can find current efforts on accuracy improvement by modulation of the laser beam assisted by an external electro-optic modulator (EOM). Thus, providing synchronization to the detection stage a resolution of few nanometers was reported in [4]. Novel algorithm proposals for a robust information extraction [5] provide a solution to discriminate the error arising from the different reflective index of the materials under measure and their media.

Recently, the use of terahertz quantum cascade lasers [6], as replacement of the conventionally used laser diodes (LD) [3], has demonstrated the capability to measure target displacements through opaque materials. In addition, innovative application fields like monitoring of independent displacement of individual portions in a surface has been shown by the use of a pulsed fiber laser in an ablation drilling process [7]. As well as a prototype for biomedical usage [8] in which an algorithm to estimate the motion of a human chest wall has been conceived with the objective of being reliable in the presence of SM signal fading.

The classical configuration is presented in Fig.1(a): one part of the emitted beam is back-reflected by the target's surface

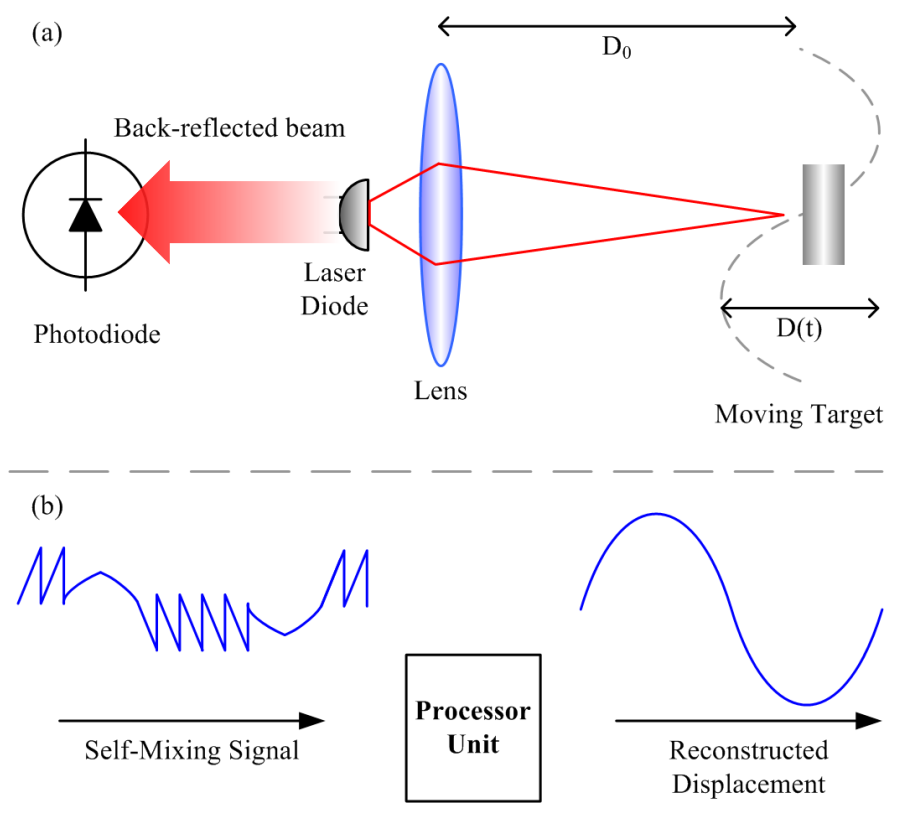

Fig. 1. (a) Self-mixing setup and (b) Displacement reconstruction representation.

into the active cavity of LD. SM signal is received by a photodiode placed in the same package as the laser source.

The measured optical output power (OOP) corresponding to the target displacement can be modeled by [3]

$$
P(t)=P_{0}\left[1+m \cdot F\left[\frac{4 \pi D(t)}{\lambda_{F}(t)}\right]\right] .
$$

Where $P_{0}$ is the emitted optical power from the laser without back-reflection, $m$ is the modulation index for the laser diode, $D(t)$ is the target displacement, $\lambda_{\mathrm{F}}$ is the emission wavelength subject to optical feedback, and $F$ is a cosine function related to the feedback coupling factor (C), used to characterize the shape of the signal when the internal oscillation of the laser is affected [2]. The sawtooth like form of the SM signal [Fig.1(b)], allows estimating the displacement of the target with a precision inferior to a half-wavelength.

In order to dimension a system for a real time application, a time constraint must be imposed based on the requirements specification. Besides, the algorithm characterization provides knowledge of the needed resources for the target architecture. In the classical displacement reconstruction algorithm [3], 
the optimization procedure launched to reach more precision works over a full signal acquisition and requires several cycles to converge. Hence, this algorithm is not suitable for real time implementation, although their main objective was to enlighten the precision reached with their method.

The technique proposed by [4] doesn't involve complicated calculations and asserts an improved resolution of few nanometers. While this could meet a timing constraint, no further analysis was performed in that direction. Moreover, the fact of adding optical components makes harder to reach the promise of producing a low cost microsystem. The elaboration depicted on [5] advises a reconstruction based on the well known SM model, this allows estimating the feedback coupling factor in a fast iterative algorithm. However the study is oriented to the robustness achieved, and the portability to an autonomous sensor remains a field to explore.

In this paper we propose to approach the prototyping of real time SM displacement sensors by distinguishing three main categories of treatments: information extraction, correction of inherent errors, and displacement reconstruction. This aims to be able to properly model such systems by analyzing and adapting the different treatments from literature so an optimal sensor for a given application could be synthesized.

Particularly we are concerned by the treatments to be performed for the sake of an accurate displacement reconstruction once that SM sawtooth like signals are detected. The focus is on an efficient implementation and a high degree of maintainability allowing a straightforward calculation of resources for a given application. The intention is to couple our algorithm to the efforts in other research areas of SM analysis like the fringe loss, speckle, or the abrupt change of $\mathrm{C}$ factor. The prototype described here is a contribution to get a step closer in the creation of a robust autonomous sensor based on this technique. The implemented algorithm is inspired from the well known First-Order-Hold (FOH) interpolation used in some Digital-to-Analog Converters (DAC) [9].

\section{THE RECONSTRUCTION PROCESS}

\section{A. Formulation}

By looking at the fringes of a measured SM sawtooth like signal [3], it can be interpreted that the detected transitions belong to an irregular sampling of the calculated displacement; hence a pure DAC interpolation is not feasible due to aliasing phenomenon. While sophisticated reconstruction methods from non uniform samples are an active research in signal processing [10], they are mainly based on the use of timeinvariant low pass filtering. To privilege the simplicity of the calculations, the essence of the integrator mechanism, which is also present in DAC interpolators, is kept as the heart of this algorithm.

Consider the transition pair (TP) composed by the present and previous detected transitions $\operatorname{tr}(N)$ and $\operatorname{tr}(N-1)$ respectively, where $N=0,1,2, \ldots$ is the number of accumulated fringes detected, and $T$ the number of samples counted between them. TP can be represented as $[\operatorname{tr}(N-1) \operatorname{tr}(N)]$.

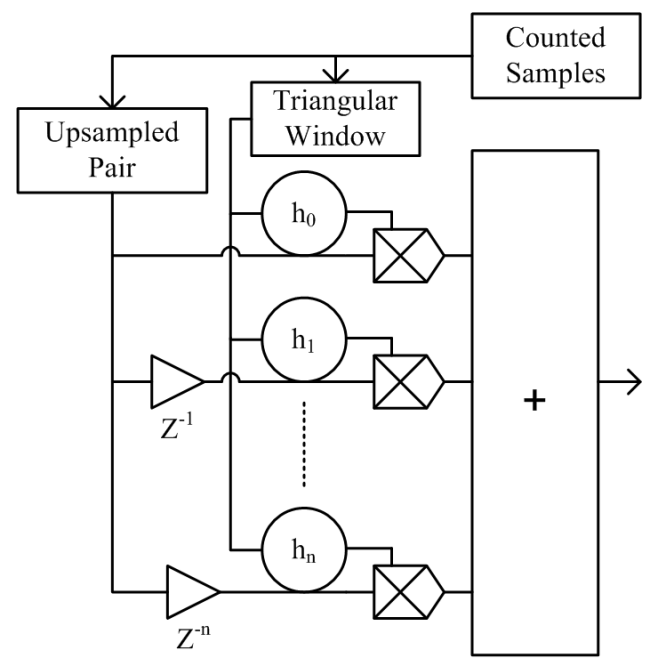

Fig. 2. Convolution in the $\mathrm{FOH}$ interpolator.

The signal $x(n)$ to convolve, is the resulting vector of upsampling TP by the factor $T$ as denoted by expression . On every new fringe arrival, TP is updated as well as the T factor.

$$
x(n)= \begin{cases}T P(n / T), & n=1,2, \ldots, T \\ 0 & \text { otherwise. }\end{cases}
$$

The convolution kernel $h(t)$ gets a size $2 T$ as established by Shannon's sampling theorem [9]. Its shape is a normalized non-causal triangular window (TRI), corresponding to the impulse response of a delayed $\mathrm{FOH}$ interpolator

$$
h(t)=(1 / T) T R I((t-T) / T) .
$$

The convolution of (2) and (3), results in the interpolated segment $\left(\mathrm{x}_{\mathrm{FOH}}\right)$ denoted by

$$
x_{F O H}(t)=\sum_{n=0}^{T} x(n) h(t-n) .
$$

From literature [2] [3], it is known that target displacement can be calculated by

$$
D(t)=N \frac{\lambda_{0}}{2}+\varepsilon
$$

where $\lambda_{0}$ is the laser diode wavelength without back-reflection, and $\varepsilon$ is the excess fringe $\left(<\lambda_{0} / 2\right)$. Since the interpolated segments keep track of the accumulated transitions, the reconstructed displacement for our algorithm is

$$
D(t)=x_{F O H}(t) \frac{\lambda_{0}}{2} .
$$

Fig.2 illustrates this method: the number of samples counted between two transitions, triggers the generation of a normalized triangular window as well as the segment bounded by the past and present transition. Both of them are convolved in order to obtain the interpolated segment which finally will 


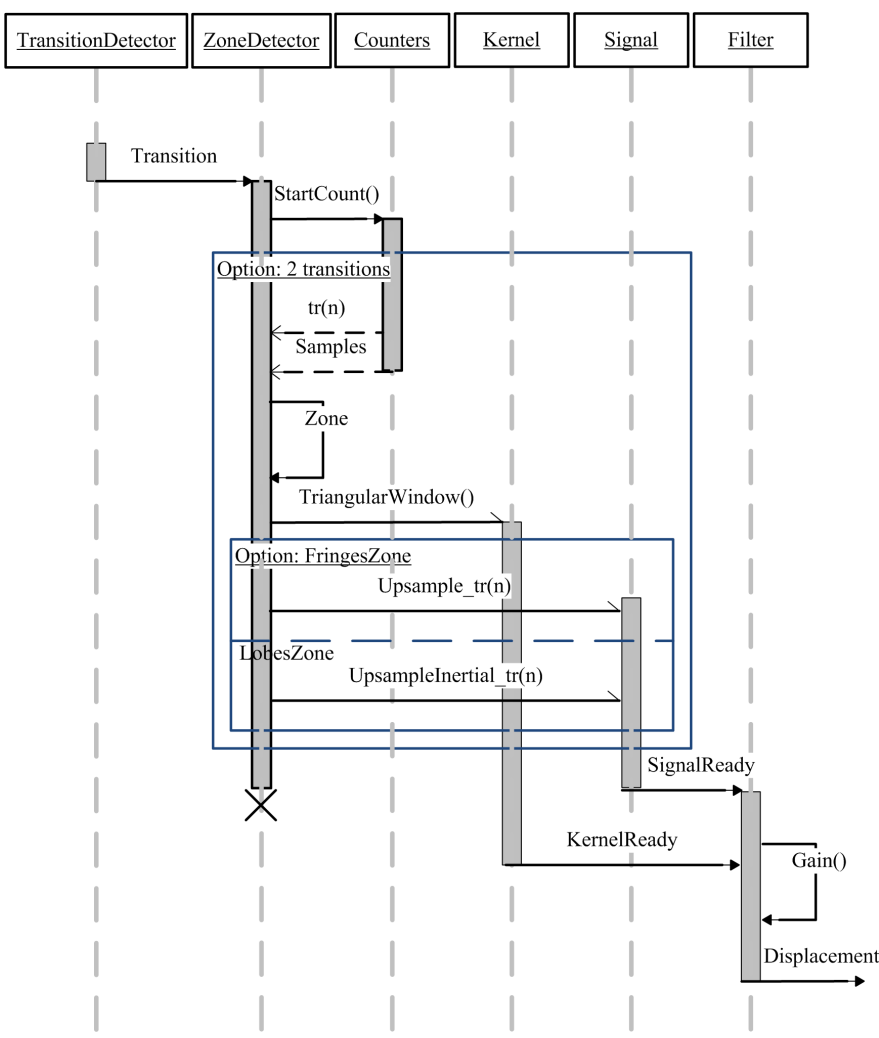

Fig. 3. Sequence diagram of the implemented algorithm

be weighted by half of the laser wavelength providing the measurement of the target displacement.

\section{B. Algorithm Implementation}

In order to satisfy embedded real time constraints, the algorithm is designed with focus on memory consumption and calculation time. The convolution kernel generation requires the arrival of two transitions and the counted samples between them. It follows that displacement calculation time is bounded by the detection of a new transition. This leads to think the worst case scenario that may happen when the analog SM signal is noisy: to detect contiguous transitions at each instant of the sampling rate. It means that the algorithm must calculate as fast as the sampling frequency for that condition and provide an easy estimation of calculation cycles for different counted samples between a pair of transitions detected. Therefore the proposed methodology highlights the critical areas susceptible of parallelism and hardware partitioning to reach this hard real time constraint.

From the sequence diagram (Fig.3), it can be seen that the analog SM signal is sampled and fed into a transition detector module. This entity is an active process over the time of measurement. It informs if the transition detected is negative or positive by implementing a high pass filter and a threshold comparison.

The zone detector stage launches the counter of samples when a transition is signaled by an interruption signal. When the counter reaches two transitions it is restarted to zero and gives an acknowledge message to the zone detector which will then distinguish two different zones of the SM signal based on the sign of the transition's pair. It will affect to the fringes zone if the sign of transitions are the same. Otherwise it will consider them as belonging to the lobe, which corresponds to " $\varepsilon$ " (positive or negative) of the SM signal.

The kernel module is asynchronously driven by the zone detector unit. It will generate the impulse response $\mathrm{h}(\mathrm{t})$ by programming (3).

Signal generation stated by (2) is asynchronous as well, and relies on the values of the accumulated present and previous transitions.

Since kernel generation is not dependent of the zero padded signal vector to be convolved, these two tasks can be implemented in parallel to increase performance. However both vectors must be synchronized when calling the filter module in order to keep coherent calculated segments.

Once that the convolved segment has been calculated, it is affected sample by sample by the gain factor $\lambda_{0} / 2$, and can be sent out to a DAC to recover the analog reconstructed displacement.

The heart of the algorithm is the convolution which remains as the most expensive process to mitigate, but it is also one of the most well understood algorithms in signal processing domain. In consequence, optimized implementations [11] can be analyzed according to particular needs.

Memory usage is also a concern in embedded sensor design. The algorithm memory consumption is directly proportional to the sampling frequency. The frequency of the displacement signals under measure is thus, also limited by the memory available in the system. In our implementation only three data buffers are required: the vector storing the calculated triangular window, the upsampled vector from the transition pair, and the one used to keep the calculated segment. The size of these buffers is determined by the counted samples between two transitions. This allows the correct prevision of resources, again, according to desired performance.

\section{Simulation Results}

Prior to the real time implementation, we validated the relevance and precision of the algorithm. Fig.4(a), shows a modeled SM signal from a laser diode with $\lambda_{0}=785 \mathrm{~nm}, \mathrm{C}$ $=3$ and normalized amplitude. The transitions presented in Fig.4(b) are calculated by threshold comparison as previously explained. It can be noticed an average number of 30 samples between detected transitions in the fringes zone and 180 samples for the lobe sections. The reconstructed signal is presented in Fig.4(c) alongside the target displacement whose amplitude is $6 \lambda_{0}=4.71 \mu \mathrm{m}$ of a sinusoidal trajectory.

From the sample to sample difference [Fig.4(d)], the maximum error of about $200 \mathrm{~nm}$ shows that the algorithm doesn't follow a perfectly smooth sinusoidal displacement. This is due to the inherent segment by segment treatments. However in displacement measurement we are mainly concerned by the amplitude of the reconstructed signal as they provide us the 
(a)

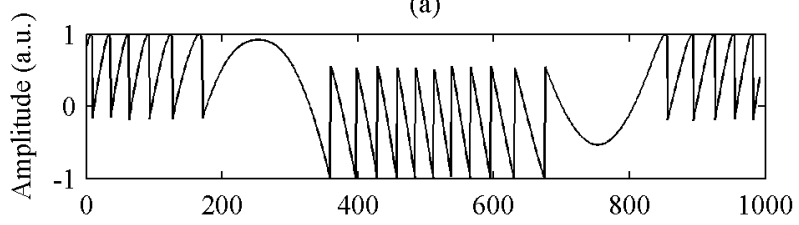

(b)

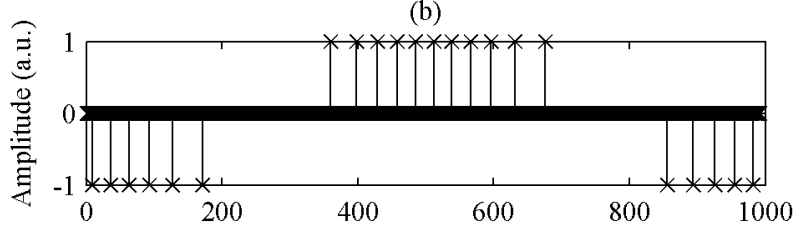

(c)

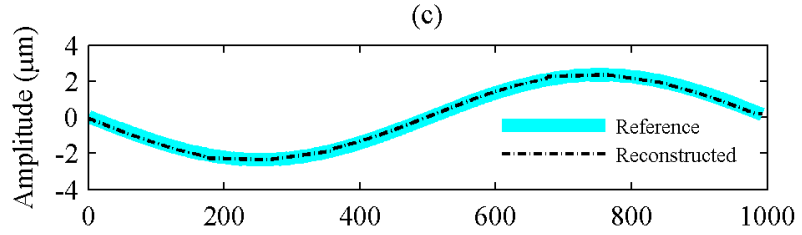

(d)

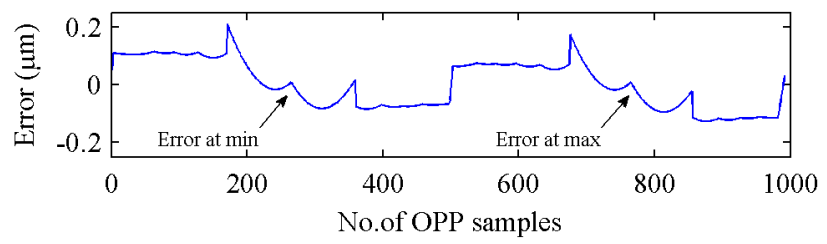

Fig. 4. (a) Modeled self-mixing signal, (b) Detected transitions, (c) Reconstructed and reference signal, and (d) Error against the original displacement.

displacement information of the target. The obtained accuracy in amplitude is $10 \mathrm{~nm}$ for this sample signal.

\section{SYSTEM VALIDATION}

The algorithm has been implemented on an off-the-shelf development board, comporting a TMS320C6416, 16-bit fixedpoint digital signal processor (DSP) with performance up to 5760 million instructions per second (MIPS) at clock rate of $720 \mathrm{MHz}$ and average instruction cycle time of 1,67 ns. Other than the calculation speed and the available optimized libraries for intensive signal processing calculations, the choice was motivated by the fact that this board offers an ADC with sampling rate from $8 \mathrm{KHz}$ to $96 \mathrm{KHz}$ and enough external memory to analyze the compromise of resources concerned face to the behavior of real environments.

We have used a Tektronix AFG3022 function generator, loaded with a modeled SM signal of the same characteristics as in the simulation to validate coherence in the results and evaluate performance of the algorithms. The frequency and amplitude are changed in real time to explore the behavior of dynamic scenarios.

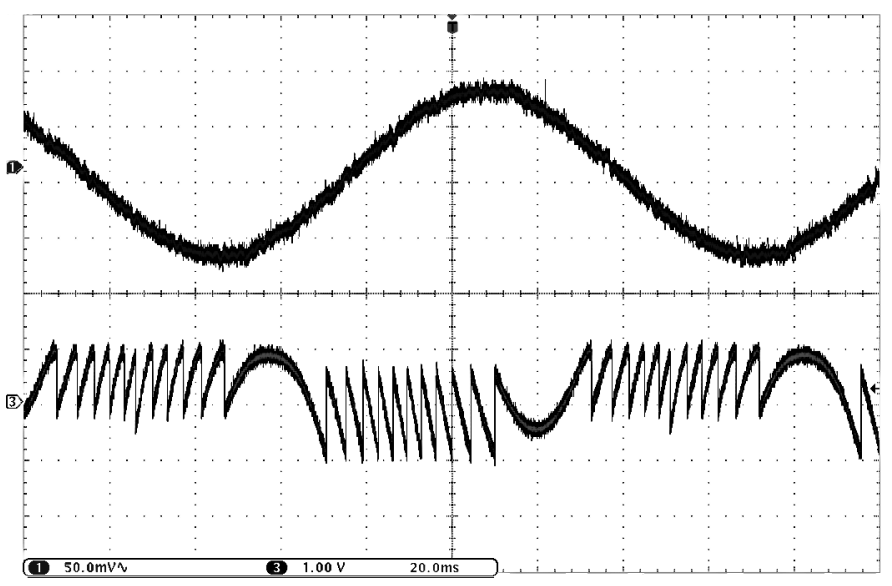

Fig. 5. Displacement reconstruction on top of the experimental SM signal.

With the profiler tool provided by the development suite for the DSP, we measure the execution time required to calculate a displacement segment from two transitions. This allows us to estimate the maximum sampling frequency authorized by the algorithm and therefore establish the maximum frequency of the target movement that our system can detect. Due to the shape of SM signals, we use a rule of thumb for the sampling rate factor of 1000 times the frequency of the modeled signal.

From Fig.5 it can be seen that signal reconstruction of $8 \mathrm{~Hz}$ is achieved properly. The measured contribution of each of the detected transitions into the DAC is close to $14 \mathrm{mV}$, while the observed peak to peak amplitude is around $160 \mathrm{mV}$. Since each transition gain is equal to $\lambda_{0} / 2$, the reconstructed amplitude can be expressed as the $6 \lambda_{0}$ of the original amplitude shown in the simulation.

For a sampling rate of $8 \mathrm{KHz}$, the average time used to calculate the segments in the fringe zone is around $20 \mu \mathrm{s}$. In the lobe zone it takes around $260 \mu$ s. Considering the sample rate of $125 \mu \mathrm{s}$ the imposed time constraints are close to $4 \mathrm{~ms}$ and $22 \mathrm{~ms}$ respectively. Thereby, for this particular SM signal the treatments are largely respecting the real time requirement. By increasing the sampling frequency in the same scenario the time to calculate a segment is linearly increased. To surpass the $4 \mathrm{~ms}$ constraint the algorithm should treat more than 6000 samples per segment, so the system is limited to $1.6 \mathrm{MHz}$ as maximum sampling frequency.

The convolution algorithm implemented has a complexity $O\left(n^{2}\right)$. However, if we are required to calculate displacements for greater frequency we can easily replace the algorithm for a circular convolution to reach a complexity $O(n \log n)$, or concentrate our efforts in a parallelized implementation for an even greater frequency.

This implementation is a proof of concept for the control of system resources in the displacement reconstruction task. The perspective is to use the DSP board to measure real target displacements and observe the reconstruction for different environment configurations. 


\section{CONCLUSION}

The implementation of the measurement system on a DSP board demonstrates the feasibility of embedded optical sensors by SM interferometry for general purpose displacement calculations. The presented methodology reduces the problem of signal reconstruction to the efficient usage of an adaptive filter, driven by the number of samples separating two transitions. We advance a straightforward estimation of the resources needed to synthesize a given application. In terms of precision, the system is in accordance to the classical reported works and could profit of recent research to be improved, notably in terms of robustness against external noise that can be introduced in real scenarios.

Besides the ease of estimation of system resources, the key point of this work is the control of the algorithm complexity to be adapted for harder real time constraints by modifying just one block of the reconstruction chain. Furthermore, this algorithm can be coupled to other signal treatments to analyze other phenomena related to the laser physics, which limit the apparition of robust SM sensors.

\section{REFERENCES}

[1] S. Donati, M. Norgia, and G. Giuliani, "A review of self-mixing techniques for sensing applications," in Lasers and Electro-Optics Society, 2004. LEOS 2004. The 17th Annual Meeting of the IEEE, vol. 1, nov. 2004, pp. 260 - 261 Vol.1.

[2] T. Bosch, C. Bes, L. Scalise, and G. Plantier, "Optical feedback interferometry," in Encyclopedia of Sensors. American Scientific Publishers, 2006, vol. 7, pp. 107-126.
[3] C. Bes, G. Plantier, and T. Bosch, "Displacement measurements using a self-mixing laser diode under moderate feedback," Instrumentation and Measurement, IEEE Transactions on, vol. 55, no. 4, pp. $1101-1105$, aug. 2006.

[4] D. M. Guo, "Quadrature demodulation technique for self-mixing interferometry displacement sensor," Optics Communications, vol. 284 no. 24, pp. 5766-5769, dec. 2011.

[5] Y. L. Fan, Y. G. Yu, J. T. Xi, and C. J. F., "Improving the measurement performance for a self-mixing interferometry-based displacement sensing system," Applied Optics, vol. 50, no. 26, pp. 5064-5072, sep. 2011.

[6] Y. L. Lim, P. Dean, M. Nikolic, R. Kliese, S. P. Khanna, M. Lachab, A. Valavanis, D. Indjin, Z. Ikonic, P. Harrison, E. H. Linfield, A. G. Davies, S. J. Wilson, and A. D. Rakic, "Demonstration of a self-mixing displacement sensor based on terahertz quantum cascade lasers," Applied Physics Letters, vol. 99, no. 8, aug. 2011.

[7] F. P. Mezzapesa, L. Columbo, M. Brambilla, M. Dabbicco, A. Ancona, T. Sibillano, F. De Lucia, P. M. Lugara, and G. Scamarcio, "Simultaneous measurement of multiple target displacements by self-mixing interferometry in a single laser diode," Optics Express, vol. 19, no. 17, pp. 16160-16173, aug. 2011.

[8] I. Milesi, M. Norgia, P. Pompilio, C. Svelto, and R. Dellaca, "Measurement of local chest wall displacement by a custom self-mixing laser interferometer," Instrumentation and Measurement, IEEE Transactions on, vol. 60, no. 8, pp. $2894-2901$, aug. 2011.

[9] J. G. Proakis and D. G. Manolakis, Digital Signal Processing - Principles, Algorithms and Applications. Pearson Prentice Hall, 2007.

[10] S. Maymon and A. Oppenheim, "Sinc interpolation of nonuniform samples," Signal Processing, IEEE Transactions on, vol. 59, no. 10, pp. $4745-4758$, oct. 2011.

[11] C. Cheng and K. Parhi, "Hardware efficient fast parallel fir filter structures based on iterated short convolution," Circuits and Systems I: Regular Papers, IEEE Transactions on, vol. 51, no. 8, pp. $1492-$ 1500, aug. 2004. 